\title{
The relationship between the nutritional status, body-mass index of patients with chronic obstructive pulmonary disease and respiratory failure and their 1-year survival
}

\author{
ำMurat Ylldız' ${ }^{1}$ DDeniz Çelik ${ }^{2}$ \\ ${ }^{1}$ Health Sciences University, Atatürk Chest Diseases and Thoracic Surgery Education and Research Hospital, Pulmonary Intensive Care Unit, \\ Ankara, Turkey \\ ${ }^{2}$ Alanya Alaaddin Keykubat University, Faculty of Medicine, Department of Pulmonology, Antalya, Turkey
}

Cite this article as: Yıldız M, Çelik D. The relationship between the nutritional status, body-mass index of patients with chronic obstructive pulmonary disease and respiratory failure and their 1-year survival. J Health Sci Med 2022; 5(1): 54-61.

\begin{abstract}
Aim: We aimed to determine whether chronic obstructive pulmonary disease (COPD) and respiratory failure patients characteristics can be defined as additional criteria to Body Mass Index (BMI), Nutritional Risk Screening (NRS-2002), and Albumin affecting the 1-year mortality.

Material and Method: One hundred eighty-sixes patients who have been hospitalized in the Pulmonary Intensive Care Unit between 01.01.2019 and 31.12.2019 were included in our study.

Results: The study comprised 186 patients and $63.5 \%$ of them were male $(n=118)$ and $36.5 \%$ were female $(n=68)$. The 1-year mortality of the patients after discharge was evaluated in two groups: those who died within 1 year $(n=87,46.7 \%)$ and the survivors' group $(n=99,53.3 \%)$. We found a significant difference between the survivors and the deceased patients in terms of weight, nutrition score, number of stays in the hospital, number of readmissions to the emergency service after discharge, and NRS-2002 score $(\mathrm{p}<0.05)$. Cox regression analysis revealed that the number of stays in the hospital, NRS-2002 score, and $\mathrm{C}$-Reactive Protein $(\mathrm{CRP})$ variables significantly affect the survival of the patients $(\mathrm{p}<0.05)$. All patients were divided into two groups (NRS-2002<4 vs. NRS-2002 $\geq 4$ ) according to the median value of NRS-2002. Thus, the survival analysis of two different groups was compared as a risk group and a high-risk group in terms of nutritional status. There was a statistically significant difference between the NRS-2002 groups in terms of survival times. The survival time of the cases in the NRS-2002 score $\geq 4$ group was significantly lower than the cases in the NRS-2002 score $<4$ group.

Conclusion: We demonstrated that NRS-2002, CRP, and prolonged stay in the hospital have a relationship with the increased mortality risk. Combining NRS-2002 score $\geq 4$ with elevated CRP levels at admission, may produce more accurate results in evaluating a patient's nutritional status in clinical practice and help make predictions about the patient's prognosis. More studies may evaluate the nutritional status of COPD patients, not only in hospitals but also in outpatient clinics.
\end{abstract}

Keywords: NRS-2002, COPD, respiratory failure, mortality

\section{INTRODUCTION}

Screening of nutritional risk has long been recommended by national and international associations. Nutritional risk has been defined to a great extent in patients with chronic obstructive pulmonary disease (COPD), and its prevalence $(20-45 \%)$ varies depending on which screening tool is used (1). It has been suggested that if malnutrition is detected by the Nutritional Risk Screening (NRS-2002) screening tool in patients hospitalized with the diagnosis of acute bronchitis, COPD exacerbation, community-acquired pneumonia, these are associated with the worsening of the disease and is directly related to mortality Providing nutritional support to these patients has a positive effect on mortality (2). However, skeletal muscle weakness and loss of fat-free body mass (FFM) are coexist with COPD (3).

Nutritional risk screening (NRS-2002) tool was developed by the Danish Association for Parenteral and Enteral Nutrition. It has also been recommended for use by the European Society for Clinical Nutrition and Metabolism (ESPEN). A patient with a total score of 3 is considered to be "at nutritional risk" (1). In the PubMed search (NRS 2002 and COPD; search date 19.10.2021), there were 9 studies. Two of them were not relevant. In these 7 studies, 
only one of them was included patients with COPD and Respiratory failure. In their study, Chen et al. (7) included COPD patients with respiratory failure. They found that Body Mass Index (BMI) and serum albumin values independently predicted in-hospital mortality. BMI and NRS-2002 predicted 1-year mortality, and it was determined that all three methods (BMI, NRS-2002, and Albumin) predicted rehospitalization within the 30 days after the discharge. Our study will be the second study with the same diagnosis and we aimed to find an additional criterion in predicting the 1-year mortality.

Our hospital is a tertiary chest diseases hospital and our pulmonary intensive care unit is specialized in COPD patients who developed acute or acute-on-chronic respiratory failure. Respiratory failure occurs in the end stage of COPD. Frequent exacerbations are common and patients are in a 'never-ending inflammation' process. Inflammation triggers the catabolic process. We observed that our patients were mostly malnourished and the prognosis of these patients was worst. The nutritional habits are mostly cultural and the way of expressing is subjective. This study comprised patients with only COPD and respiratory failure in a specific ICU.

We hypothesized that a single, simple and easy screening tool could be effective to predict malnutrition earlier in this patient population.

We aimed to determine the COPD and respiratory failure patient's characteristics as an additional criterion to BMI, NRS-2002 score, and albumin levels in predicting the 1 -year mortality.

\section{MATERIAL AND METHOD}

The study was carried out with the permission of Health Sciences University Keçiören Education and Training Hospital Clinical Studies Ethics Board (Date: 14.09.2021 Decision No: 2012-KAEK-15/2355). All procedures were carried out in accordance with the ethical rules and the principles of the Declaration of Helsinki.

\section{Selection of Patients}

One-hundred eighty-six patients who have hospitalized in the Pulmonary Intensive Care Unit between 01.01.2019 and 31.12.2019 were included in our study. All patients whose data were available and who gave consent for clinical studies at admission were included in the study. The study design is cross-sectional.

\section{Inclusion Criteria}

All COPD and respiratory patients hospitalized in the pulmonary intensive care unit (ICU) have been included in the study. For obtaining real-life data, the patients who had comorbidities didn't exclude. The patients with COPD were diagnosed and treated by a pulmonologist before and had received inhalation therapies for a long period. All patients admitted to the Emergency room of our hospital. Our hospital is a tertiary chest diseases hospital and the Emergency room team consists of pulmonologists and pulmonology residents. All patients' primary diagnosis was COPD exacerbations and/or decompensation of respiratory failure. The indications of pulmonary ICU hospitalizations were either severe exacerbation and/ or decompensation of respiratory failure. All patients' Global Initiative for Chronic Obstructive Lung Disease (GOLD) classifications consisted of group D.

\section{Exclusion Criteria}

We planned to perform post-discharge survival analysis, so we excluded only malignancy patients. The patients who died in the hospital (who could not be discharged) were excluded too.

\section{Nutritional Risk Screening-2002 (NRS-2002)}

NRS-2002 is a screening system developed by Kondrup et al. (8) based on the retrospective analysis of randomized controlled trials. NRS-2002 was recommended by the ESPEN and was developed for malnutrition risk screening in hospitalized patients within the first 48 hours following hospitalization. The Turkish validation study of the scale was done by Bolayır in 2014 (9). Nutrition screening and evaluation were done using the three-component of the NRS-2002 screening tool. The first component evaluates the nutritional status with three separate items. The nutritional status' is scored between $0-3$ points. The first component consists of the following: BMI category $(<20.5$ or $>20.5$ ), weight loss category [ $>5 \%$ at 3 months, $>5 \%$ at 2 months and $>5 \%$ at 1 month (>15\% at 3 months)], and decreased food intake as a proportion of normal need in the previous week $(0 \% 25,25-50 \%, 50-75 \%$ and $>75 \%)$. If the answer to any of these three questions is yes, the second stage of the scoring system is started.

The second and third components assess disease severity and age, respectively. The disease severity is scored between 0-3 points. 1 point includes patients with chronic illnesses who have been hospitalized for complications. These patients are debilitated but may get out of the bed regularly. Protein requirement is increased, but is mainly at a level that can be overcome with oral diet and supplementation. 2 point represents patients who have become bedridden due to infection or major abdominal surgery as a prototype. Protein requirements are markedly increased and in most cases, artificial feeding may be required. 3 points consist of patients in need of intensive care, under inotropic or ventilatory support as a prototype. Finally, all patients over the age of 70 receive an additional score increase. If the total score is 3 or more; the patient is at nutritional risk and a nutritional plan is initiated. If the score is $<3$; should be scanned once 
a week. If there is a major surgical operation planned, a nutrition plan should also be developed. The BMI cut-off value for malnutrition diagnosis is $18.5 \mathrm{~kg} / \mathrm{m}^{2}(8)$.

\section{Statistical Method}

Analyzes were made with the Statistical Package for the Social Sciences (SPSS) Statistics 26 package program. When analyzing the data, the frequencies (number, percentage) were given for categorical variables; while descriptive statistics (mean, standard deviation) were given for numerical variables. Normality assumptions of numerical variables were examined with the Kolmogorov Smirnov normality test and it was observed that the variables were normally distributed. For this reason, parametric statistical methods were used in the study. The differences between the two independent groups were analyzed using the Unpaired T-Test. Relations between two categorical variables were checked with Chi-Square analysis. Fisher's Exact Test was used when the expected value assumption was not provided in the Chi-Square analysis. The survival duration of the patients was examined with Kaplan Meier analysis, and variables affecting survival were checked with Cox Regression analysis. Statistical significance in the analyzes was interpreted at the level of 0.05 for the p-value.

The study was designed as cross-sectional and analyzed patients hospitalized whole 1 year. In the retrospective power analysis for the adequacy of the survival analysis, at a significance level of 0.05 and an effect width of 0.8 ; the power of the study was calculated as $97.3 \%$.

\section{RESULTS}

The study comprised 186 patients and $63.5 \%$ of them were male $(n=118)$ and $36.5 \%$ were female $(n=68)$. The 1 -year mortality of the patients after discharge was evaluated in two groups: those who died within 1 year after discharge $(\mathrm{n}=87,46.7 \%)$ and the survivors' group $(\mathrm{n}=99,53.3 \%)$ (Table 1).

The relation between the comorbidities and mortality was analyzed. A statistically significant relationship was found between the presence of Acute or Chronic Renal Disease and mortality $(\mathrm{p}=0.016)$.

\section{Table 1. Conditions and comorbidities that affect mortality}

\begin{tabular}{|c|c|c|c|c|c|c|}
\hline & & \multicolumn{2}{|c|}{ Survivor group $(n=99)$} & \multicolumn{2}{|c|}{ Exitus group $(n=87)$} & \multirow[b]{2}{*}{$\mathbf{p}$} \\
\hline & & $\mathbf{n}$ & $\%$ & n & $\%$ & \\
\hline \multirow{2}{*}{ Gender } & Female & 31 & 45.6 & 37 & 54.4 & \multirow{2}{*}{0.113} \\
\hline & Male & 68 & 57.6 & 50 & 42.4 & \\
\hline Age (year & & \multicolumn{2}{|c|}{$67.00 \pm 12.43$} & \multicolumn{2}{|c|}{$74.30 \pm 11.82$} & $0.000^{*}$ \\
\hline \multicolumn{2}{|c|}{ Readmission to the hospital after discharge } & 30 & 53.6 & 26 & 46.4 & 0.951 \\
\hline \multicolumn{2}{|c|}{ Readmission to the emergency room within 30 days after discharge } & 13 & 52.0 & 12 & 48.0 & 0.895 \\
\hline \multicolumn{2}{|c|}{ Readmission to the hospital within 30 days after discharge } & 9 & 52.9 & 8 & 47.1 & 0.980 \\
\hline \multicolumn{2}{|c|}{ Domiciliary long term oxygen therapy } & 90 & 55.9 & 71 & 44.1 & 0.064 \\
\hline \multicolumn{2}{|c|}{ Domiciliary noninvasive mechanical ventilation } & 39 & 54.9 & 32 & 45.1 & 0.714 \\
\hline \multicolumn{2}{|c|}{ Bronchiectasis } & 10 & 76.9 & 3 & 23.1 & 0.076 \\
\hline \multicolumn{2}{|c|}{ Pulmonary hypertension } & 6 & 66.7 & 3 & 33.3 & 0.506 \\
\hline \multicolumn{2}{|c|}{ Sleep apnea or obesity hypoventilation syndrome } & 6 & 66.7 & 3 & 33.3 & 0.506 \\
\hline \multicolumn{2}{|c|}{ Central nerveus system diseases } & 7 & 41.2 & 10 & 58.8 & 0.296 \\
\hline \multicolumn{2}{|c|}{ Decompensated respiratory acidosis } & 6 & 50.0 & 6 & 50.0 & 0.817 \\
\hline \multicolumn{2}{|c|}{ Acute bronchitis } & 5 & 55.6 & 4 & 44.4 & 1.000 \\
\hline \multicolumn{2}{|c|}{ Bening prostate hyperplasia } & 7 & 63.6 & 4 & 36.4 & 0.476 \\
\hline \multicolumn{2}{|c|}{ Pulmonary sequelae due to tuberculosis } & 7 & 70.0 & 3 & 30.0 & 0.341 \\
\hline \multicolumn{2}{|c|}{ Other metabolic diseases } & 6 & 75.0 & 2 & 25.0 & 0.287 \\
\hline \multicolumn{2}{|c|}{ Pleurisy } & 1 & 20.0 & 4 & 80.0 & 0.187 \\
\hline \multicolumn{2}{|c|}{ Rheumatic diseases } & 1 & 33.3 & 2 & 66.7 & 0.600 \\
\hline \multicolumn{2}{|c|}{ Pneumonia } & 20 & 42.6 & 27 & 57.4 & 0.090 \\
\hline \multicolumn{2}{|c|}{ Pulmonary thromboembolism } & 10 & 41.7 & 14 & 58.3 & 0.224 \\
\hline \multicolumn{2}{|c|}{ Restrictive lung disease } & 12 & 70.6 & 5 & 29.4 & 0.132 \\
\hline \multicolumn{2}{|c|}{ Diabetes mellitus } & 22 & 55.0 & 18 & 45.0 & 0.800 \\
\hline \multicolumn{2}{|c|}{ Congestive heart failure } & 20 & 44.4 & 25 & 55.6 & 0.175 \\
\hline \multicolumn{2}{|c|}{ Coronary artery disease } & 16 & 48.5 & 17 & 51.5 & 0.547 \\
\hline \multicolumn{2}{|c|}{ Acute or chronic renal disease } & 2 & 18.2 & 9 & 81.8 & $0.016^{*}$ \\
\hline Atrial fib & & 6 & 35.3 & 11 & 64.7 & 0.120 \\
\hline Hyperten & & 43 & 58.9 & 30 & 41.1 & 0.212 \\
\hline Hypoxic & & 15 & 55.6 & 12 & 44.4 & 0.793 \\
\hline Hypercap & ilure & 57 & 58.8 & 40 & 41.2 & 0.114 \\
\hline
\end{tabular}


There were no significant differences between the survivors and the exitus group in terms of gender, rehospitalization after discharge, presence of NRS-2002 itself and, its scoring.

We found a statistically significant difference between the survivors and the deceased patients in terms of weight, nutrition score, number of stays in the hospital, number of readmissions to the emergency service after discharge, and NRS-2002 score $(\mathrm{p}<0.05)$ (Table 2).

\begin{tabular}{|c|c|c|c|c|c|}
\hline & \multicolumn{2}{|c|}{$\begin{array}{c}\text { Survivor } \\
\text { group }(n=99)\end{array}$} & \multicolumn{2}{|c|}{$\begin{array}{c}\text { Exitus group } \\
(\mathbf{n}=\mathbf{8 7})\end{array}$} & \multirow{2}{*}{ p } \\
\hline & Mean & SD & Mean & SD & \\
\hline Height & 165.06 & 9.74 & 163.29 & 7.85 & 0.178 \\
\hline Weight & 75.41 & 17.87 & 69.79 & 18.60 & $0.038^{*}$ \\
\hline Body mass index & 27.83 & 7.05 & 26.23 & 6.96 & 0.124 \\
\hline Disease severity score & 2.61 & 0.79 & 2.67 & 0.74 & 0.593 \\
\hline Nutrition score & 0.40 & 0.55 & 0.57 & 0.62 & $0.049^{*}$ \\
\hline NRS 2002 score & 3.36 & 1.16 & 3.93 & 1.07 & $0.001^{*}$ \\
\hline $\begin{array}{l}\text { Number of stay in } \\
\text { hospital (day) }\end{array}$ & 11.69 & 6.64 & 17.48 & 13.26 & $0.000^{*}$ \\
\hline $\begin{array}{l}\text { Number of } \\
\text { hospitalizations after } \\
\text { discharge }(n=56)\end{array}$ & 1.70 & 0.46 & 1.70 & 0.46 & 0.951 \\
\hline $\begin{array}{l}\text { Number of admissions } \\
\text { to the emergency room } \\
\text { after discharge }(n=90)\end{array}$ & 1.44 & 0.50 & 1.60 & 0.49 & $0.037^{\star}$ \\
\hline $\begin{array}{l}\text { COPD diagnosis } \\
\text { duration (year) }\end{array}$ & 6.06 & 6.32 & 5.83 & 6.19 & 0.800 \\
\hline
\end{tabular}

The weight of the exitus group was significantly less than the survivors. In addition, the nutritional score, the number of stays in the hospital, the number of readmissions to the emergency service after discharge, and the NRS-2002 score levels of the exitus group are significantly higher than the survivors.

The relationship between the laboratory values and mortality was presented in Table 3.

\begin{tabular}{|lccccc|}
\hline $\begin{array}{l}\text { Table 3. Descriptive statistics of laboratory measurements by } \\
\text { mortality status }\end{array}$ & $\begin{array}{c}\text { Survivor group } \\
\text { (n=99) }\end{array}$ & $\begin{array}{c}\text { Exitus group } \\
\text { (n=87) }\end{array}$ & p \\
\cline { 2 - 5 } & Mean & SD & Mean & SD & \\
\hline Blood urea nitrogen & 20.65 & 10.78 & 26.30 & 15.37 & $0.004^{*}$ \\
Uric acid & 5.58 & 2.04 & 6.14 & 2.44 & 0.093 \\
Albumin & 34.74 & 4.66 & 33.69 & 4.97 & 0.137 \\
Hemoglobin & 14.38 & 2.53 & 13.26 & 2.47 & $0.003^{*}$ \\
Creatinine & 0.95 & 0.39 & 1.12 & 0.86 & 0.085 \\
C-reactive protein & 57.72 & 69.87 & 85.98 & 104.75 & $0.034^{*}$ \\
Total protein & 62.79 & 6.18 & 62.29 & 7.40 & 0.615 \\
Hematocrit & 46.86 & 8.16 & 43.22 & 8.20 & $0.003^{*}$ \\
White blood cell & 11047.84 & 4691.13 & 11970.46 & 6759.97 & 0.276 \\
APACHE-II score & 12.27 & 4.42 & 13.65 & 4.49 & $0.037^{*}$ \\
\hline $\begin{array}{l}\text { A: Unpaired T-Test, *: p<0.05, APACHE-II: Acute Physiologic Assessment and Chronic } \\
\text { Health Evaluation II, SD: Standard deviation }\end{array}$
\end{tabular}

There is a statistically significant difference between the survivors and the exitus group in terms of Blood Urea Nitrogen (BUN), Hemoglobin, C-Reactive Protein (CRP), Hematocrit, Acute Physiologic Assessment and Chronic Health Evaluation II (APACHE II) scores $(p<0.05)$. While the hemoglobin and hematocrit levels of the survivors were significantly higher; the BUN, CRP, APACHE II scores of the exitus group were significantly higher than those of the survivors (Table 3).

\section{Determination of the Variables Affecting Survival}

Variables affecting the mortality of patients were included in the Cox Regression model as an independent variable. The variables included in the model were readmission to the emergency room after discharge, weight, nutritional score, number of stays in the hospital, NRS-2002 score, acute or chronic renal disease status, BUN, Hemoglobin, CRP, Hematocrit, and APACHE II score. Among these variables, the assumption of risk invariance for the categories of readmission to the emergency room after discharge and acute or chronic renal disease status, which are categorical variables, was analyzed graphically and the variables were considered appropriate to be included in the model. The absence of a high correlation between independent variables, which is another important assumption of Cox regression, was analyzed from the correlation table of the Cox Regression Analysis; and high correlations were observed between NRS-2002 score and nutritional score and between Hemoglobin and Hematocrit. Therefore, nutritional score and Hematocrit variables were excluded from the model. Later, the model was run by utilizing the Backward Wald method. When the results were examined, it was understood that the most suitable model was established in 6 steps and weight, hemoglobin level, APACHE II score, and acute or renal disease variables were excluded from the model. The results for the remaining variables are shown in Table 4.

When Table 4 is examined, it can be seen that after the exclusion of the variables, the number of stays in the hospital, NRS-2002 score, BUN, and CRP variables remained in the model. When the Omnibus test results are examined, the established Cox Regression model is statistically significant $(p<0.05)$. When the coefficients of the independent variables in the model are examined, it can be inferred that the number of stays in the hospital, NRS-2002 score, and CRP variables significantly affect the survival of the patients statistically $(\mathrm{p}<0.05)$. As a result, when the number of stays in the hospital increases by 1 unit, the risk of death increases by 1.036 times. When the NRS-2002 score increases by 1 unit, the risk of death increases by 1.337 times. When CRP increases by 1 unit, the risk of death increases by 1.002 times. 


\begin{tabular}{|lcccccc|}
\hline & B & $\begin{array}{c}\text { Standard } \\
\text { Error }\end{array}$ & $\mathbf{p}$ & Exp(B) & \multicolumn{2}{c|}{ \%95 Confidence Interval Exp (B) } \\
\cline { 5 - 7 } & & & & & Lower Bound & Upper Bound \\
\hline Number of stay in the hospital (days) & 0.036 & 0.009 & $0.000^{*}$ & 1.036 & 1.019 & 1.054 \\
NRS-2002 score & 0.290 & 0.105 & $0.006^{*}$ & 1.337 & 1.088 & 1.643 \\
Blood urea nitrogen & 0.015 & 0.008 & 0.054 & 1.015 & 1.000 & 1.030 \\
C-reactive protein & 0.002 & 0.001 & $0.034^{*}$ & 1.002 & 1.000 & 1.005 \\
\hline Omnibus Test Chi Square $=47.186 \mathrm{p}=0.000$ & & & & & & \\
\hline
\end{tabular}

NRS-2002 scores were naturally high in both groups due to the high risk of malnutrition in the patient groups $(3.36 \pm 1.16$ vs $3.93 \pm 1.07)$. In our study, the vast majority of patients were elderly, and the fact that the patients were hospitalized in the intensive care unit. All patients were divided into two groups (NRS-2002 $<4$ vs NRS-2002 $\geq 4$ ) according to the median value of NRS-2002. Thus, the survival analysis of two different groups was compared as a risk group and a high-risk group in terms of nutritional status (Table 5).

\begin{tabular}{|c|c|c|c|c|}
\hline \multirow{2}{*}{$\begin{array}{l}\text { NRS-2002 } \\
\text { score }\end{array}$} & \multirow{2}{*}{$\begin{array}{c}\text { Survival } \\
\text { Period } \\
\text { (days) }\end{array}$} & \multicolumn{2}{|c|}{ Survival Rate } & \multirow{2}{*}{$\begin{array}{l}\text { Total Case } \\
\text { Number }\end{array}$} \\
\hline & & Estimate & $\begin{array}{c}\text { Standard } \\
\text { Error }\end{array}$ & \\
\hline \multirow{3}{*}{$\begin{array}{l}\text { NRS-2002<4 } \\
(\mathrm{n}=71)\end{array}$} & 90 & 0.873 & 0.039 & 9 \\
\hline & 180 & 0.859 & 0.041 & 10 \\
\hline & 365 & 0.746 & 0.052 & 18 \\
\hline \multirow{3}{*}{$\begin{array}{l}\text { NRS-2002 } \geq 4 \\
(n=115)\end{array}$} & 90 & 0.739 & 0.041 & 30 \\
\hline & 180 & 0.643 & 0.045 & 41 \\
\hline & 365 & 0.557 & 0.046 & 51 \\
\hline
\end{tabular}

As a result of Kaplan Meier analysis, the patients who have the NRS-2002 score $<4$, the 90 -day survival rate is $87.3 \%$, the 180 -day survival rate is $85.9 \%$, and the 1 -year survival rate is $74.6 \%$. the patients who have the NRS2002 score $\geq 4$, the 90 -day survival rate is $73.9 \%$, the 180 day survival rate is $64.3 \%$, and the 1-year survival rate is $55.7 \%$.

The survival time of the patients in the NRS-2002 $<4$ group was 602,769 days, while the patients in the NRS$2002 \geq 4$ group were 422,767 days (Table 6). There was a significant difference between the NRS-2002 groups in terms of survival times. The survival time of the patients in the NRS-2002<4 group was significantly higher than the patients in the NRS-2002 $\geq 4$ group $(\mathrm{p}<0.001)$. Figure 1 shows survival analysis according to NRS-2002 groups

In a summary, in this specific group of patients with COPD and respiratory failure, the patients have been divided into two groups as survivors and exitus groups in 1-year follow-up after discharge. Gender, weight, rehospitalization after discharge, presence of domiciliary LTOT, or domiciliary NIV were not significant between the two groups. Nutrition score, BUN, Hemoglobin, CRP, Hematocrit, APACHE II scores, number of

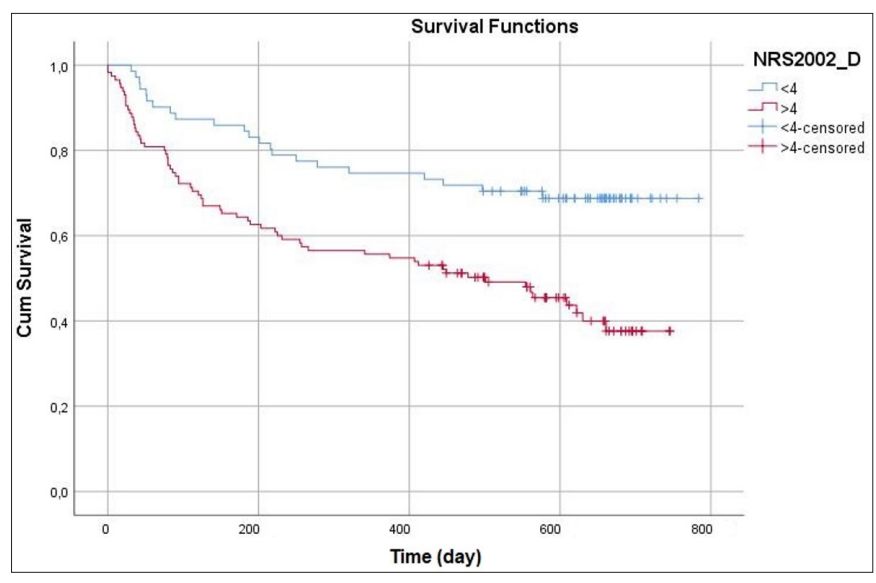

Figure 1. Survival analysis according to NRS-2002 groups.

stays in the hospital, number of readmissions to the emergency service after discharge, and NRS-2002 score were significant between the two groups. There was a significant relationship between the presence of Acute or Chronic Renal Disease and mortality.

Variables affecting the mortality of patients were included in the Cox Regression model, each one as an independent variable. The variables included in the model were readmission to the emergency room after the discharge, weight, nutritional score, number of stays in the hospital, NRS-2002 score, acute or renal disease status, BUN, Hemoglobin, CRP, Hematocrit, and APACHE II score. The most suitable model was established in 6 steps and weight, hemoglobin, APACHE II score, and acute or renal disease variables were excluded from the model. The number of stays in the hospital, NRS-2002 score, BUN, and CRP variables remained in the model. The number of stays in the hospital, NRS-2002 score, and CRP variables significantly affect the survival of the patients $(\mathrm{p}<0.05)$. All patients were divided into two groups (NRS-2002<4 vs NRS-2002 $\geq 4$ ) according to the median value of NRS2002. Thus, the survival analysis of two different groups was compared as a risk group and a high-risk group in terms of nutritional status. There was a significant difference between the NRS-2002 groups in terms of survival times. The survival time of the patients in the NRS-2002 score $\geq 4$ group was significantly lower than the patients in the NRS-2002 score $<4$ group. 


\begin{tabular}{|c|c|c|c|c|c|c|c|c|}
\hline \multirow{2}{*}{$\begin{array}{l}\text { NRS-2002 } \\
\text { score }\end{array}$} & \multicolumn{2}{|r|}{ Mean } & \multicolumn{2}{|c|}{ 95\% Confidental Interval } & \multicolumn{2}{|c|}{ Median } & \multicolumn{2}{|c|}{ 95\% Confidental Interval } \\
\hline & Survival & Standard Error & Lower Limit & Upper Limit & Survival & Standard Error & Lower Limit & Upper Limit \\
\hline$<4$ & 602.769 & 33.703 & 536.71 & 668.827 & . & . & & . \\
\hline$\geq 4$ & 422.767 & 28.782 & 366.354 & 479.181 & 501 & 105.589 & 294.045 & 707.955 \\
\hline
\end{tabular}

\section{DISCUSSION}

COPD is characterized by progressive chronic systemic inflammation with frequent exacerbations, and malnutrition is common in the clinical course. Many factors, especially smoking and systemic inflammation induce TNF- $\alpha$ and neutrophils, which may cause malnutrition despite adequate nutrient intake (10). Therefore, the presence of malnutrition in COPD patients may play an important role in disease progression and symptom control. Various methods have been used to screen the presence of malnutrition in COPD patients (10). In addition, while in most of the previous studies analyzing the nutritional status of COPD patients other nutritional risk scales were used, we evaluated the nutritional status of the patients with the NRS-2002 scale in our study. As a result of the study, we found that if the NRS-2002 score is $<4$, the 90 -day survival rate is $87.3 \%$, the 180 -day survival rate is $85.9 \%$, and the 1 -year survival rate is $74.6 \%$. In the patients with an NRS-2002 score $\geq 4$, the 90 -day survival rate is $73.9 \%$, the 180 -day survival rate is $64.3 \%$, and the 1 -year survival rate is $55.7 \%$. The survival time of the cases in the NRS-2002 $<4$ group was significantly higher than the cases in the NRS-2002 $\geq 4$ group

In their study, Ogan et al. (11) found a significant correlation between NRS-2002 malnutrition risk assessment score and the mMRC (Modified Medical Research Council) dyspnea scale in the Global Initiative for Chronic Obstructive Lung Disease (GOLD) C and D group patients with stable COPD, and it was determined that there was a relationship between symptom level and nutritional status of patients with COPD. In the study of Benedik et al. (12) it was observed that malnourished COPD patients had low upper arm and middle arm circumference. According to the literature, as the nutritional status deteriorated, a decrease is observed around the upper and middle arm, and the lowest values were found in patients with the highest risk of malnutrition.

Marco et al. (13) evaluated nutritional deficiencies of 118 COPD patients without exacerbation and/or who were not hospitalized according to the ESPEN definition, and included parameters such as involuntary weight loss, BMI, and fat-free mass index (FFMI). The prevalence of malnutrition was observed to be $24.6 \%$ and it was found that an increase in malnutrition was associated with the increased risk of mortality.
In their study, Chen et al. (14) found that both BMI and NRS-2002 scores were independent predictors of 1-year mortality in COPD patients with respiratory failure, but the NRS-2002 score was superior to BMI for the prediction of 1-year mortality. In the ROC analysis, they revealed that the cutoff value that can predict long-term mortality is 3 points and above for NRS-2002. It has been inferred that when the NRS-2002 score is $\geq 3$ during admission, a COPD patient with respiratory failure is more likely to die within 1 year after discharge. In our study, the mean NRS-2002 score of the survivors was $3.36 \pm 1.16$, while that of the exitus group was $3.93 \pm 1.07$.

We found a significant difference between the survivors and the exitus group in terms of weight, nutritional score, number of stays in the hospital, and NRS-2002 score. Evaluation of the nutritional status of COPD patients by NRS-2002 can provide information about age and disease severity compared to BMI. Thus, NRS-2002 can comprehensively show the nutritional status of COPD patients with respiratory failure and help accurately predict their long-term prognosis.

Baumgartner et al. (15) investigated the effects of individualized nutritional support on mortality and other important clinical outcomes in a subgroup of patients with lower respiratory tract infections. They found a 25\% reduction in 30-day mortality with nutritional support in patients with lower respiratory tract infections who were at nutritional risk. Evidence on nutritional support is insufficient in patients with the infection without critical illness. However, several publications are showing that nutritional support has positive effects on outcomes for patients at risk, in the general patient population $(16,17)$.

CRP has been used for many years (18). In the study by $\mathrm{Qu}$ et al. (19) CRP levels of nonsurvivors in ICU were found to be significantly higher than those of survivors. As a dichotomous variant, $\mathrm{CRP}>62.8 \mathrm{mg} / \mathrm{L}$ at admission to ICU was associated with increased ICU mortality, regardless of whether the patient was septic or not. This may be related to the lack of specificity of CRP whether the patient has sepsis or not (20). Although a study claims that the assessment of serum CRP levels is not an adequate test to predict mortality in ICU patients (21), other studies showed that CRP is a suitable predictor of mortality $(22,23)$. In our study, there was a significant difference in terms of CRP values between the survivor group and the exitus group in the 1-year mortality assessment. Also, we found significantly lower CRP 
values in survivors (mean CRP $57.72 \mathrm{mg} / \mathrm{L}$ in survivors, and $85.98 \mathrm{mg} / \mathrm{L}$ in the exitus group). In our study, it was observed that the risk of death increased 1.002 times when CRP increased by 1 unit. Therefore, in clinical practice, CRP may be useful to help clinicians assess the prognosis of ICU patients.

In our study, there is a significant difference between the survivors and the exitus groups in terms of Hemoglobin and Hematocrit values. We found that the hemoglobin and hematocrit levels of the survivors were significantly higher than the exitus group.

In a study conducted by Gammelager et al. (24) in 2012, it was found that $15 \%$ of the patients had acute kidney damage during admission to the ICU. An increase by two times in 30-day mortality was identified in patients who were in the acute kidney injury group during admission to the ICU. Relative mortality in patients with acute kidney injury was found to be associated with $33-64 \%$ increased mortality in the 31-365 days following the admission to the ICU. The relationship between comorbidities and mortality was investigated in our study, there was a statistically significant relationship between Acute or Chronic renal disease and mortality. In addition, BUN values were found to be significantly higher in the exitus group. In all large studies involving large numbers of ICU patients, an increase in short/medium term mortality, and in-hospital mortality for patients with acute kidney injury during admission to the ICU or during their stay in the ICU was reported. In these studies, the relative risk of in-hospital mortality increased by 1-1.6 times for patients with acute kidney injury and between 1.6 and 4.1 times for patients with acute renal failure $(25,26)$.

Scoring systems [such as APACHE II; Simplified Acute Physiology Score II (SAPS II) and Multiple Organ Dysfunction Score (MODS)] are used to determine the severity of the disease, response to treatment, expected mortality rates, and intensive care performance of patients followed up in intensive care units (27). In their study comparing the worst APACHE II scores at first admission and in the first 24 hours, Ho et al. (28) found that the expected mortality was $15.5 \%$ and $19.3 \%$ respectively, and the actual mortality was $16.3 \%$. They stated that the sensitivity and specificity of the APACHE II score determined at the first admission were decent and that it could be used for intensive care patients without trauma. We evaluated the patients with the APACHE II scoring system during their admission to the ICU. In line with the literature, we found a significant difference between the APACHE II scores and the number of admissions to the ICU after discharge between the two groups. The number of admissions to the emergency room after discharge and APACHE II score levels of the exitus group were significantly higher than those of the survivors. Studies are suggesting that the discharge APACHE II score' outperforms the 'admission APACHE II score, for readmission to the hospital (29).

\section{Limitations}

Our study was carried out in a single center and designed as a retrospective. In addition, the patients were evaluated with a single nutritional assessment method at admission. The NRS-2002 score is the nutritional risk screening tool recommended by ESPEN and is more suitable than most other available methods due to its simplicity, applicability, and non-invasive nature. However, as NRS-2002 contains subjective content, the outcome of the evaluation is somewhat dependent on the evaluators. For this reason, we consider that an experienced evaluator is needed. The strong aspect of our study is, the NRS-2002 scoring was applied to the patients by experienced dietitians and intensive care physicians to avoid subjectivity and bias. Also, The COPD exacerbations are closely affected by seasonal changes. We include patients in 1 year to avoid seasonal bias.

\section{CONCLUSION}

We demonstrated that high NRS-2002 scores, high CRP levels, and prolonged stay in the hospital were associated with the mortality risk. Combining the NRS-2002 tool with CRP and prolonged stay in the hospital can produce more accurate results in evaluating a patient's nutritional status in clinical practice and help make predictions about the patient's prognosis. More studies may evaluate the nutritional status of COPD patients, not only in hospitals but also in outpatient clinics.

\section{ETHICAL DECLARATIONS}

Ethics Committee Approval: The study was carried out with the permission of Health Sciences University Keçiören Education and Training Hospital Clinical Studies Ethics Board (Date: 14.09.2021 Decision No: 2012-KAEK-15/2355).

Informed Consent: Because the study was designed retrospectively, no written informed consent form was obtained from patients.

Referee Evaluation Process: Externally peer-reviewed.

Conflict of Interest Statement: The authors have no conflicts of interest to declare.

Financial Disclosure: The authors declared that this study has received no financial support.

Author Contributions: All of the authors declare that they have all participated in the design, execution, and analysis of the paper, and that they have approved the final version. 


\section{REFERENCES}

1. Ingadottir AR, Beck AM, Baldwin C, et al. Two components of the new ESPEN diagnostic criteria for malnutrition are independent predictors of lung function in hospitalized patients with chronic obstructive pulmonary disease (COPD). Clin Nutr 2018; 37: 1323-31.

2. Baumgartner A, Hasenboehler F, Cantone J, et al. Effect of nutritional support in patients with lower respiratory tract infection: Secondary analysis of a randomized clinical trial. Clin Nutr 2021; 40: 1843-50.

3. Engelen MP, Schols AM, Does JD, Wouters EF. Skeletal muscle weakness is associated with wasting of extremity fat-free mass but not with airflow obstruction in patients with chronic obstructive pulmonary disease. Am J Clin Nutr 2000; 71: 733-8.

4. Chen R, Xing L, You C, Ou X. Prediction of prognosis in chronic obstructive pulmonary disease patients with respiratory failure: A comparison of three nutritional assessment methods. Eur J Intern Med 2018; 57: 70-5.

5. Kondrup J, Allison SP, Elia M, et al. Educational and Clinical Practice Committee, European Society of Parenteral and Enteral Nutrition (ESPEN). ESPEN guidelines for nutrition screening 2002. Clin Nutr 2003; 22: 415-21.

6. Bolayır, B. Hospitalize Hastalarda Nutrisyonel Değerlendirme Testi NRS-2002'nin (Nutritional Risk Screening-2002) geçerlilik ve güvenilirliğinin değerlendirilmesi. phD Thesis, Hacettepe University Faculty of Medicine, Department of Internal Medicine, Ankara; 2014.

7. Lainscak M, Benedik B, Farkas J, et al. Mini nutritional assessment, body composition, and hospitalizations in patients with chronic obstructive pulmonary disease. Respir Med 2011; 105: 38-43.

8. Ogan N, Yıldırım F, Süzen B, Baha A, Akpınar EE. Does nutritional risk screening 2002 correlate with the dyspnea status of patients with GOLD stage C-D chronic obstructive pulmonary disease? Turk Thorac J 2020; 21: 49-53.

9. Benedik B, Farkas J, Kosnik M, et al. Mini nutritional assessment, body composition, and hospitalizations in patients with chronic obstructive pulmonary disease. Respir Med 2011; 105: 38-43.

10.Marco E, Sánchez-Rodríguez D, Dávalos-Yerovi VN, et al. Malnutrition according to ESPEN consensus predicts hospitalizations and long-term mortality in rehabilitation patients with stable chronic obstructive pulmonary disease. Clin Nutr 2018; 38: 2180-6.

11. Chen R, Xing L, You C, Ou X. Prediction of prognosis in chronic obstructive pulmonary disease patients with respiratory failure: A comparison of three nutritional assessment methods. Eur J Intern Med 2018; 57: 70-5.

12. Reber E, Gomes F, Vasiloglou MF, Schuetz P, Stanga Z. Nutritional Risk Screening and Assessment. J Clin Med 2019; 8: 1065.

13. Felder S, Braun N, Stanga Z, et al. Unraveling the link between malnutrition and adverse clinical outcomes: association of acute and chronic malnutrition measures with blood biomarkers from different pathophysiological states. Ann Nutr Metab 2016; 68: 164-72.

14. Cederholm T, Bosaeus I, Barazzoni R, et al. Diagnostic criteria for malnutrition - An ESPEN Consensus Statement. Clin Nutr 2015; 34: 335-40.

15.Schmit X, Vincent JL. The time course of blood C-reactive protein concentrations in relation to the response to initial antimicrobial therapy in patients with sepsis. Infection 2008;36(3):213-9

16. Qu R, Hu L, Ling Y, et al. C-reactive protein concentration as a risk predictor of mortality in intensive care unit: a multicenter, prospective, observational study. BMC Anesthesiol 2020; 20: 292.

17. Clyne B, Olshaker JS. The C-reactive protein. J Emerg Med 1999; 17: 1019-25.
18. Silvestre J, Póvoa P, Coelho L, et al. Is C-reactive protein a good prognostic marker in septic patients?. Intensive Care Med 2009; 35: 909-13.

19.Lobo SM, Lobo FR, Bota DP, et al. C-reactive protein levels correlate with mortality and organ failure in critically ill patients. Chest 2003; 123: 2043-9.

20.Prieto MF, Kilstein J, Bagilet D, Pezzotto SM. C-reactive protein as a marker of mortality in intensive care unit. Med Intensiva 2008; 32: 424-30.

21.GamGammelager H, Christiansen CF, Johansen MB, Tønnesen E, Jespersen B, Sørensen HT. One-year mortality among Danish intensive care patients with acute kidney injury: a cohort study. Crit Care 2012; 16: 124.

22. Hoste EA, Clermont G, Kersten A, et al. RIFLE criteria for acute kidney injury are associated with hospital mortality in critically ill patients: a cohort analysis. Crit Care 2006; 10: 73.

23. Bagshaw SM, George C, Dinu I, Bellomo R. A multi-center evaluation of the RIFLE criteria for early acute kidney injury in critically ill patients. Nephrol Dial Transplant 2008; 23: 1203-10

24.Bouch D, Thompson J. Severity scoring systems in the critical ill. Continuing Education in Anesthesia and Critical Care 2008; 8:181-5.

25.Ho K, Dobb G, Knuiman M, Finn J, Lee K, Webb S. A comparison of admission and worst 24-hour Acute Physiology and Chronic Health Evaluation II scores in predicting hospital mortality a retrospective cohort study. Crit Care 2006; 10: 4

26. Lee H, Lim CW, Hong HP, et al. Efficacy of the APACHE II score at ICU discharge in predicting post-ICU mortality and ICU readmission in critically ill surgical patients. Anaesth Intensive Care 2015; 43: 175-86. 